\title{
MobiCon: Mobile Video Recording with Integrated Annotations and DRM
}

\author{
J. Lahti, K. Pentikousis, and M. Palola \\ VTT, Technical Research Centre of Finland \\ Kaitoväylä 1, 90571 Oulu, Finland
}

\begin{abstract}
The proliferation of camera phones alongside the deployment of high data rate wireless networks will rapidly increase the amount of digital video content. Efficient content management tools will be essential for the evolution of mobile multimedia services. We introduce MobiCon, a mobile phone application, which combines meta-content descriptions and Digital Rights Management (DRM) seamlessly. With MobiCon, users can capture digital videos, annotate them using an ontology tree and free text entries, upload them to a remote video server, choose which clips to share and with whom, and take advantage of standards-based DRM to control the entire life-cycle of each video. Once stored on a video server, clips are indexed facilitating later retrieval. We report on field trial results with real users, which show that MobiCon is rather easy to learn and use, even for inexperienced users, and present insights from user comments on DRM, video annotation, and uploading.
\end{abstract}

\section{INTRODUCTION}

Modern camera phones can record videos of up to several minutes, depending on the amount of memory available. Such videos are not stored permanently on the mobile device (UE): users want to add the videos to their collection, and share them with friends and relatives. Some will even opt for making part of their digital content available from a web site, or add it to their blog entries. For example, Lifeblog is an application that enables users to maintain a multimedia "diary" using a mobile phone by amalgamating images, videos, notes, and text messages (nokia.com/lifeblog). Nevertheless, when considering videos alone, it has certain drawbacks. First, Lifeblog uses a two-step process, which requires a PC: users must transfer videos via USB and Bluetooth to the PC and then upload them to a remote server-they cannot share them directly with other people or store them on a remote server using their UE. Second, a small set of metadata can be added, but not in a standards-compliant format.

On the other hand, Vodafone K. K. Movie Sha-mail subscribers can capture relatively short videos, and e-mail them as attachments over the cellular network (www.vodafone.jp). This form of video e-mail is quite popular, but does not support video annotation. Furthermore, clips cannot be delivered to more than five recipients simultaneously, and they are not permanently accessible from a server.

We anticipate that user-created videos will soon dominate media content and propose to combine the advantages of the aforementioned (and other similar) applications in one, allowing for personalized mobile video creation and management, as well as scalable distribution of the video content to different terminals. The application ought to be easy to learn and use, and should let the user create content with the UE without a need for post-processing on a workstation. Users should maintain full control of the entire video management chain, from the source to the target device, by tapping on a digital rights management (DRM) framework. DRM is an umbrella term for a set of technologies developed to protect against unauthorized copying and distribution of copyrighted material [1]. DRM is usually associated with the entertainment industry intellectual property rights (IPR), but it can be used to define content usage rules too. For example, the Open Mobile Alliance (www.openmobilealliance.org) designed the OMA DRM standard, which despite the limited set of content management methods, is well suited for the mobile environment.

To facilitate video retrieval, the application should be pluggable to a searchable repository, as it is extremely timeconsuming and inefficient to find videos using filenames only. Sarvas et al. [2], for example, present a system for creating metadata for images originating from mobile devices and claim that effective management of media content is not possible without metadata. They propose leveraging contextual metadata at the point of capture to address problems of semantic and sensory gaps. The evaluated video application, MobiCon, combines point-of-capture metadata annotations and DRM parameters with a video delivery and sharing clips via the CANDELA video server [3, 4].

\section{FUNCTIONAL SPECIFICATION}

Mobile phone applications should use resources, such as network capacity, battery power, and CPU with frugality, and must not block critical events like incoming calls. Additionally small color displays for example, 176x208 pixels restrict user interface (UI) design. Applications, nevertheless, must be easy to use, rich in functionality, and appealing to the user. The main functions of MobiCon (recording, metadata annotation, storing, and sharing video clips) must adhere to these guidelines. The recording function relies on video encoding methods that dictate bandwidth, memory, and power usage and define the video clip format. As mentioned above, video metadata annotation is necessary for searching stored clips. The best time for annotating video clips is right after recording them, and both user-provided and automatically acquired can be utilized. However, the mobile phone input method (keypad) is designed for navigating menus and typing 
mainly numerals, so entering large amounts of text can be frustrating. The application should assist the user by making this process as easy as possible and collecting metadata during the video capture [2]. The focus of the collected metadata ought to be on concepts, which users can remember easily. Conversely, if the metadata are too general, most searches will generate too many results. The collected metadata along with any inferred information should be displayed in a list form to minimize typing. Video storage and video sharing connote that clips can be temporarily stored on the UE and transferred to a server for a long-term preservation and distribution. MobiCon's client-server video sharing architecture is well suited for this (Figure 1): videos and metadata captured by the UE can be uploaded to a CANDELA video server. Once the video and metadata are successfully delivered and stored on the video server, users can notify the recipient(s) about the video availability. An essential feature of video sharing is DRM support which allows the user to control for how long and how many times a recipient can play and/or redistribute a shared video clip.

Due to the limited computing and storage resources, most UEs support only a couple of standardized video content formats. Besides providing permanent and searchable storage, the video server can also run computation-intensive operations, such as video transcoding, to allow users to access stored clips several times at the most appropriate format, based on their current UE and network connection. Using standards, such as H.263 [5] and MPEG-7 [6], improves interoperability between different devices and technologies. The remaining of the paper focus on MobiCon, its video capture and annotation user interface, and the results of a field trial evaluation.

\section{MoBiCon}

We developed MobiCon [3], a Java 2 Platform, Micro Edition (J2ME) MIDlet, which implements the functional specification laid out in the previous section. MobiCon works on Symbian OS v8.0 UEs, for example the Nokia 6630. Figure 2 illustrates the MobiCon architecture, which comprises five components; video recording using the integrated phone camera; local (temporary) storage; video delivery; recipient notification via text messaging (SMS); and UI Manager.

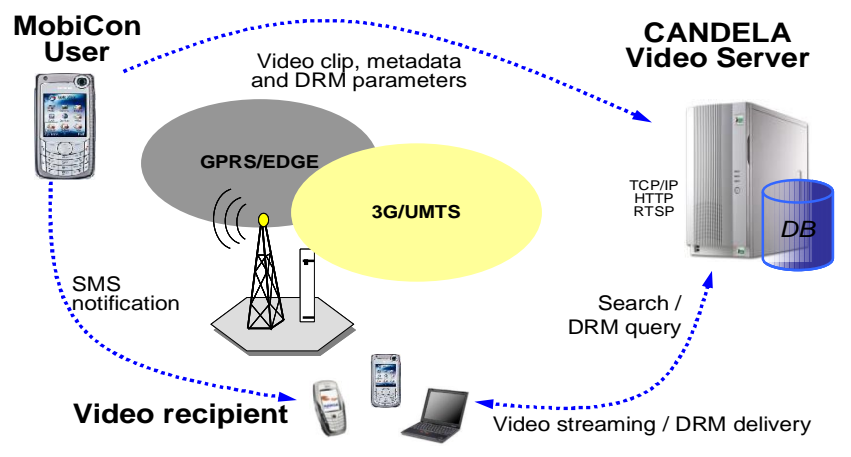

Figure 1 MobiCon video sharing architecture.

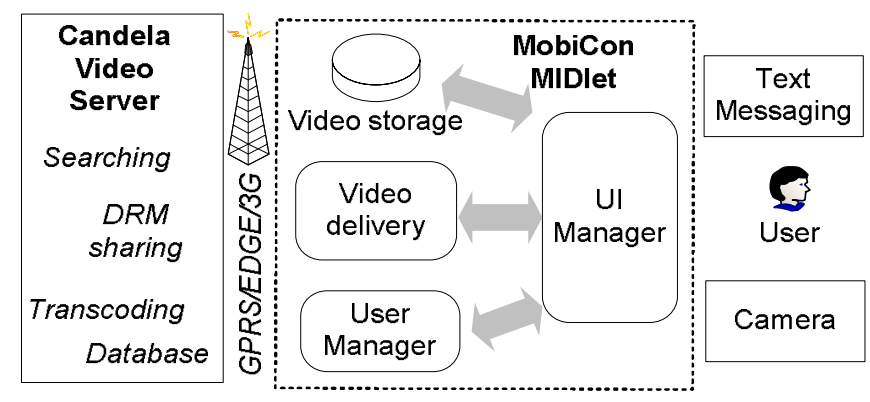

Figure 2. The MobiCon Architecture.

Two J2ME extensions, Mobile Media API 1.1 (JSR-135) and Wireless Messaging API (JSR-120), provide the underlying functionality needed for video capturing using the integrated camera and sending a video clip link as a text message, respectively. The UI Manager provides the application user interface and application logic. User Manager keeps user information such as name, address, country, user modified ontology tree, previously given annotations, and user credentials.

The captured video clip is initially stored on the mobile phone until user completes annotation and sends clip to the CANDELA video server. From the CANDELA video server the users can later access their personal home video collections through web-based user interface using advanced search mechanisms utilizing the given metadata annotations. In addition to HTML and XHTML-based user interfaces and video clip transcoding, the CANDELA video server also packages the video clips using DRM tools (forum.nokia.com/drm) and stores video clip with metadata to distributed relational database.

Figures 3 and 4 present a set of screenshots of MobiCon application in a mobile phone. Figure 3 presents the basic video capture and annotation procedures and Figure 4 shows user interfaces for DRM protected video clip preparation and sharing. After recording video clip (Screenshots 1-2), the user has the options to view, add metadata, upload, and save video clip (3). In "add metadata" view (4) the user can select previously given terms (for example "Add: Statue"), annotate the video clip with terms from an ontology tree (5-7) and add free-text descriptions (8) using the nine-button keypad. Also at (3) by selecting "3.Settings", the user can update his/hers personal information, which is automatically added to metadata descriptions for each video. When annotating using an ontology tree, the main level view (5) contains general terms, which are then expanded in the sub-level menus $(6,7)$. Users can also add new terms on the tree using keypad. Before sending the video clip and metadata to the video server, the user has an option to enable DRM protected delivery to control how video clip is used by recipients. Figure 4 shows screenshots for setting the Forward Lock delivery (9-12) for a captured clip and receiving a DRM protected video clip (1316). 


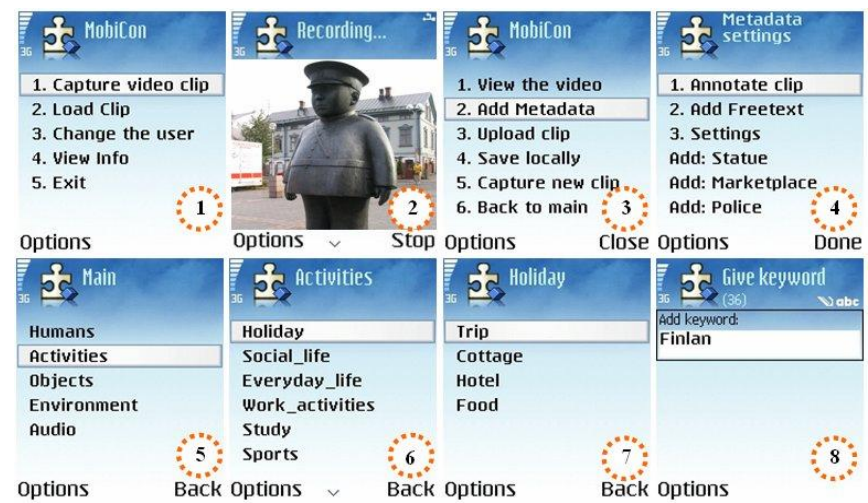

Figure 3 The MobiCon video annotation UI. The numbers in circles index different screenshots.



Figure 4 The MobiCon DRM video sharing UIs.

MobiCon currently supports only Forward Lock and Combined Delivery DRM methods. The Separate Delivery method is left for future work, if there is user interest. The user starts by selecting all recipient numbers taking advantage of the phone's contacts list (10). Then the user can specify settings for the Forward Lock feature by setting that the video can be viewed only five times (11) between September 20-22, 2005 (12). MobiCon user uploads the video clip with DRM settings and metadata in MPEG-7 format to the video server in which the video is packed to DRM format and stored to database. MobiCon also sends text messages to the selected recipients of the video clip. The text message contains the URL pointing to the protected video clip uploaded at the video server (13). After clicking the URL, the DRM-protected video is downloaded (14) to the recipients' phone where it can be displayed according to the rules specified by the MobiCon user (15). Note that the recipients do not need MobiCon to receive and view the DRM-protected videos (13-16).

\section{Evaluation Methodology}

The main goal of the MobiCon field trial was twofold: quantify application usability and assess the overall appeal of this novel multimedia service. A secondary goal was to discover any usability problems and application bugs that evaded alpha testing in the lab using Nokia 7610 and 6630 Ues.
We conducted a field trial with ten real users going on and about in the metropolitan area of Oulu, Finland, recording videos, annotating them and uploading them to our CANDELA video server over a cellular network, in a fashion similar to that illustrated in Figure 1. The trial aimed at replicating typical user behavior in a realistic environment between February 14 and March 13, 2005. All participants were given a Nokia 6630 mobile phone, which can record videos and supports the OMA DRM 1.0 specification. We installed MobiCon on the UEs before the trial started because we were not interested in evaluating the application installation phase on Symbian mobile phones. As it turned out, pre-installation shielded inexperienced users from several technical details leading, perhaps, to more favorable results.

After briefly introducing MobiCon, we let users take it for a "test ride." All participants were instructed to capture and upload as many and as long videos as they would like, but that they had to complete the trial within two weeks. Even though they did not have to perform any specific tasks, they were instructed to try all the annotation mechanisms and share videos using DRM protection.

The ten participants, aged 24-40 years, had attended higher education and three out of ten were females. We expect that this demographic group is more likely to adopt new mobile services. Eight out of ten users had prior experience with mobile services and "advanced" mobile phone operations, including web and WAP browsing, still picture capturing, and video recording. We categorized two of the participants as "inexperienced" based on their answers on a pre-trial questionnaire, which led us to anticipate that they might encounter considerable difficulties using MobiCon. Moreover, only three out of ten participants had downloaded an application over the cellular network, prior to the field trial. This concurs with our decision to pre-install MobiCon, as it might have considerably affected the overall user stance towards the application. Finally, four out of ten participants had never used WWW service to receive, for example, weather updates, stock quotes, or travel information on a mobile phone.

During the trial period, we used data logging to gather data about the performance of the application and other contextrelated data, including user details, time and date; number of UI selections made; video duration and size in bytes; session duration; type and number of metadata descriptions provided; and upload bit rate. In addition, we logged whether each video was loaded from the phone or captured in real time, whether DRM parameters were specified, and which DRM method was chosen. Captured videos were stored, along with their annotations, making it possible to derive conclusions from their type and length, and how well the respective annotations correlated with the actual content.

After the trial period, we followed up with an evaluation questionnaire in order to further assess user experiences during the field test, collect information about MobiCon's usability, detect software problems or inconsistencies, and harness improvement ideas. 
The questionnaire comprised 16 multiple-choice questions using a scale of 1-6 in decreasing affirmative order, that is, " $1=$ agree strongly; 2 = agree; $\ldots 6=$ don't know." An additional seven free-text questions for generating qualitative data were included as well. Some of the questions aimed at assessing the overall acceptability of new technologies used in MobiCon, such as DRM and video annotation.

\section{FIELD TRIAL RESUltS}

The participants recorded and successfully uploaded 132 videos during the field trial, but the variability was high. On average, it means that users captured 13.2 videos per user. The maximum number of videos captured by a single user was 17 , the minimum was 3 and the median was 9 . There is no clear correlation between the participants' previous experience and number of captured clips, but we observed that users who attended social events, such as a birthday party, during the trial period seem to have captured video clips more eagerly.



Figure 5 MobiCon usability improves considerably within ten sessions.

Figure 5 shows the average number of UI selections and the average session duration, $\bar{t}_{\text {session }}$, for each of the first ten sessions. To assess the steepness of the MobiCon learning curve and how easy is it to use it on a regular basis [7], we examined the number of choices users did and measured the time taken to complete the specific task, $t_{\text {task }}$, for every successfully completed basic usage scenario, i.e., capture a video, annotate and upload it to the CANDELA video server. Users could also specify DRM parameters, but that was left to their discretion. In order to derive a comparable metric, independent of the clip length, $t_{\text {video }}$, and the particular user, we define the user session duration, $t_{\text {session }}$, (1) as

$$
t_{\text {session }}=t_{\text {task }}-t_{\text {video }}
$$

Figure 5 indicates that, on average, the first four sessions, $\bar{t}_{\text {session }}$, can be characterized as "exploratory" as users attempt to see what can they achieve with the application, and what selections are available in the application menus.

Figure 5 also highlights that during these initial sessions, participants need almost three minutes to complete each session successfully, during which they make more than 40 UI selections, on average. In certain cases, users made errors in their choices and had to press the "Back" button. Such cases count as two user selections: one for the erroneous choice and one for the "Back" button. Trial data portend that after ten sessions only, many users will be able to claim they have "mastered" MobiCon. Even so, a common remark we received in the post-trial questionnaire was that users did not fully understand the intricacies of the two supported DRM methods (Forward Lock and Combined Delivery), despite the fact that these were explained to all participants before the field trial.

One important requirement for the developed application was to offer a mechanism for the user to add metadata to the captured videos. Adding metadata manually is very problematic in mobile devices, so in the evaluation quite a lot emphasis was put on testing how the solutions for adding metadata worked. On average, each video was annotated with 2.4 metadata terms. However, the number of terms associated with each video varied considerably between different users. Some of the participants annotated each video with more than four terms on average, while some added less than a single term. Totally, 19 out of 132 videos were not given any metadata annotations. When looking more closely to those 19 unannotated videos it can be seen that eight of them were cases where there user only wanted to share videos with somebody using DRM protected sharing methods, thus not adding the video to the database. Rest 11 un-annotated videos were divided between two users, whom for some reason just did not want to annotate their videos with extra keywords to those that were added automatically. Contrary to our expectations, we found no clear correlation between the participants' previous experience with mobile devices and services and the amount of metadata collected. On the contrary, there is a clear correlation between the type of the video content and the amount of metadata: videos that depicted real-life activities were amply annotated. Shorter videos were annotated minimally, as one might expect. From these results it can be seen that the user is willing to give metadata annotations if he/she has motivation to do it and there is a clear event to be described with the metadata.

With respect to using ontology trees vs. nine-button keypad entries to annotate the data, most users agreed that the former is a much more convenient way to add metadata to a clip. Nevertheless, many of them said that neither the ontology tree structure nor its size were ideal. In an attempt to improve this aspect of the UI, we are planning an ontology annotation mechanism which is more personalized and adaptive. Overall, the participants were satisfied with the ease of use of the application. Less experienced users generally gave lower ratings, but none of them thought that the system was "too complicated to use." Seven out of ten participants complained about "slow network connections" and four complained about the "unreliability of the network." Since most of the metropolitan Oulu area is covered by a $3 \mathrm{G}$ network, these results come as a surprise. Of course, the asymmetric nature of the 
cellular network plays an important role, because operators often opt to limit uplink data rates to $64 \mathrm{~kb} / \mathrm{s}$ ( $c f .384 \mathrm{~kb} / \mathrm{s}$ for the downlink).

UEs also play a role: the Nokia 6630, for example, cannot upload faster than $128 \mathrm{~kb} / \mathrm{s}$. Though third generation networks provide considerably faster connections than GPRS/EDGE [8], they may still not be fast enough for video uploads. Users also complained about losing captured videos due to "phone and/or application crashes." Application trace post-processing show-ed that most of these crashes could be traced to erratic key pressing and that in a couple of cases the application or phone did indeed crash.

A couple of aspects of the MobiCon, which were somewhat unsatisfactory, were the transparency of the DRM technologies and the security questions made by mobile phone. The evaluation showed that the most inexperienced test users were quite annoyed because the video clip submission view was followed a view which asked would user want to include DRM settings or not. This optional DRM feature should have been offered to users at different place on UI for example on screen shot 3 in Figure 3. Also, the selection of DRM method should have been more self-explanatory instead of selecting between Forward Lock and Combined-Delivery. A couple of the users were also confused about the numerous security questions generated by the phone's operating system. In general, when J2ME application uses mobile phone resources such as camera, the user is asked to grant that access to the application. The same also happens before SMS sending, video clip storing on the mobile phone, and before accessing the mobile network. However, some of these can be granted for application permanently via settings of mobile phone and developing a signed MIDlet.

Finally, eight out of ten users found this type of service "useful," and stated that they would be interested in using these services in the future as well. However, it is important to note that participants were not asked to pay any data transmission fees themselves. It is not certain whether pecuniary responsibility would have an effect on their eagerness to use the application in the future. Nonetheless, the results hint that there is a need for this kind of mobile video services and we anticipate that favorable billing will play a central role in wide user adoption.

\section{CONCLUSION AND FUTURE WORK}

We developed a new mobile application that allows for remote, permanent storage, efficient retrieval, and controlled delivery of video clips captured with a camera phone. The application, called MobiCon, successfully integrates the OMA DRM 1.0 specification with remote video storage and annotation using ontology trees, free text entries, and automatically gathered information. For example, time, date, user name, and address.

We evaluated MobiCon in a real-world environment and found that the majority of the users think that the service is useful, the application is not complex at all, and that it is easy to learn and use. The field trial was successful: it verified the potential of the application, identified problems, and provided excellent ideas for improvements.

Based on user comments, we also added a context-aware web service, which improves the quality of the collected metadata, for example, by translating GPS coordinates to location names and we are developing more personal and adaptive ontology annotation mechanism; therefore fulfilling better the needs of each user. Another improvement will be made to the usability of the mobile user interface, therefore more advanced and automated metadata collection methods are required. One of these better metadata collection methods could be audio annotation, so the user could add metadata descriptions to the videos just by speaking. In addition, the possibility of using other network connections, besides mobile networks, will be studied. Moreover, the remarks about slow cellular upload links, especially over GPRS, and the potential of high transfer fees in a public $3 \mathrm{G}$ network, prompted us to consider adding a peer-to-peer delivery channel as well. This is expected to satisfy a number of users who would prefer to capture several videos, annotate them on the spot, but upload them later. Finally, we note that explaining the use of DRM and clarifying its benefits to real users should play an essential role in any future field trials.

\section{ACKNOWLEDGMENT}

The work was conducted as part of the ITEA CANDELA (Content Analysis, Networked Delivery and Architectures) project during 2004-2005. Funding granted by TEKES - Tekes National Technology Agency of Finland and VTT Technical Research Centre of Finland is greatly appreciated. We thank Jari Korva from VTT and Tapio Seppänen from University of Oulu for guidance and comments.

\section{REFERENCES}

[1] W. Jonker, and J.-P. Linnartz, "Digital rights management in consumer electronics products", IEEE Signal Processing Mag., vol. 21, no. 2, March 2004, pp. 82-91.

[2] R. Sarvas, E. Herrarte, et al., "Metadata creation system for mobile images", in Proc. ACM MobiSys 2004, Boston, Massachusetts, USA, June 2004, pp. $36-48$.

[3] J. Lahti, U. Westermann, et al., "Context-Aware Mobile Capture and Sharing of Video Clips", Handbook of Research on Mobile Multimedia, I. K. Ibrahim \& J. Kepler (eds.), Idea Group, Hershey, Pennsylvania, USA, May 2006.

[4] A. Sachinopoulou, S.-M. Mäkelä, et al., "Personal video retrieval and browsing for mobile users", In in Proc. 17th International Symposium Electronic Imaging Science and Technology, San José, California, USA, 16-20 January 2005.

[5] ITU-T Recommendation H.263, "Video coding for low bit rate communication", 1998.

[6] Shih-Fu Chang, T. Sikora, and A. Purl, "Overview of the MPEG-7 standard", IEEE Transactions on Circuits and Systems for Video Technology, vol. 11, no. 6, June 2001, pp. 688-695.

[7] J. Nielsen, Usability Engineering. Academic Press, San Diego, California, USA, 1993.

[8] R. Prasad, W. Mohr, and W. Konhäuser, Third Generation Mobile Communication Systems, Artech House, Boston, Massachusetts, USA, 2000. 